\title{
The Far-Infrared Radiation in M83
}

\author{
L. V. Jones and W. C. Keel \\ University of Alabama, Dept. of Physics and Astronomy, Box 870324, \\ Tuscaloosa, AL 34587
}

\section{Introduction}

The fact that a galaxy is barred may have an effect on the source of the farinfrared (FIR) radiation observed. Yet, to date, no one has addressed the effects that that might have on interpreting the global FIR signal. We hope to add to the existing data on FIR radiation in galactic bulges by comparing a barred galaxy, M83, to an unbarred galaxy, M74.

We use optical, $\mathrm{H} \alpha$ and UV images that are blurred to the same resolution as the IRAS HIRES images. Looking at the blurred UV images, bright "globs" were chosen and apertures were fit to them. The "globs" were then located on the optical, $\mathrm{H} \alpha$ and FIR images and fluxes were determined for each aperture used.

\section{The Energy Balance}

One way to understand the physical source of the FIR radiation in spiral galaxies is to look at the energy balance. We examine the starlight seen directly versus the FIR radiated. Examining these ratios, we are able to distinguish which population dominates in the heating of the grains which, in turn, radiate in the FIR.

We use models of old and young populations to calculate scaling factors (OPSF and YPSF, respectively) which are used to convert the optical and UV flux densities measured into bolometric fluxes. The model young population is a cluster of stars that has undergone constant star formation (SF) since the beginning of the universe. The model old population is a cluster of stars that underwent a burst of SF sometime near the beginning of the universe and has had no SF since.

The very young population is represented by the $H \alpha$ flux converted into a Lyman continuum flux. The young population is represented by the UV fluxes multiplied by the YPSF. The old population is represented by the optical band fluxes multiplied by the OPSF for each band. The easiest way to see which population is the dominating heating source is to compare these fluxes to the FIR fluxes for each "glob".

\section{Analysis}

The evidence, up until now, supports the idea that FIR is coming from dust heated by an old population in M74 and M83 (see von Hippel \& Bothun 1990, 
Chen et al. 1992, Cornett et al. 1994, Bohlin et al. 1983, Bohlin, Cornett, \& Hill (1990), Fitt et al. 1992).

We expect to see a difference in the dominating heating sources in barred and unbarred galaxies. Tables 1 and 2 show the ratios for each "glob" in each galaxy. Looking at the tables, there is no obvious difference between the source of FIR in barred or unbarred galaxies.

Table 1. Globs in M74.

\begin{tabular}{lclll}
\hline $\begin{array}{c}\text { Distance } \\
\text { (arcsec) }\end{array}$ & $\begin{array}{c}\text { Old I } \\
\text { B/FIR }\end{array}$ & $\begin{array}{c}\text { Old II } \\
\text { V/FIR }\end{array}$ & $\begin{array}{c}\text { Young } \\
\text { UV/FIR }\end{array}$ & $\begin{array}{l}\text { V. Young } \\
\text { H } \alpha / \text { FIR }\end{array}$ \\
\hline 19.42 & 0.239 & 0.858 & 0.129 & 0.003 \\
177.92 & 0.433 & - & 0.246 & - \\
229.95 & 2.256 & - & 1.161 & - \\
154.34 & 0.780 & - & 0.299 & - \\
119.82 & 0.390 & 1.145 & 0.182 & - \\
96.50 & 0.311 & 0.869 & 0.157 & - \\
117.74 & 0.514 & 1.307 & 0.214 & 0.0003 \\
143.23 & 0.323 & - & 0.185 & - \\
61.05 & 0.302 & 1.029 & 0.154 & 0.002 \\
91.38 & 0.351 & 1.025 & 0.154 & -
\end{tabular}

Table 2. Globs in M83.

\begin{tabular}{lllll}
\hline $\begin{array}{c}\text { Distance } \\
\text { (arcsec) }\end{array}$ & $\begin{array}{c}\text { Old I } \\
\text { B/FIR }\end{array}$ & $\begin{array}{c}\text { Old II } \\
\text { I/FIR }\end{array}$ & $\begin{array}{c}\text { Young } \\
\text { UV/FIR }\end{array}$ & $\begin{array}{c}\text { V. Young } \\
\text { H } \alpha / \text { FIR }\end{array}$ \\
\hline & & & & \\
194.59 & 0.116 & 0.719 & 0.025 & 0.063 \\
474.52 & 4.070 & 18.491 & 0.336 & 1.826 \\
849.15 & 54.613 & 224.174 & 1.873 & - \\
318.81 & 8.389 & 35.220 & 0.341 & 2.007 \\
598.31 & 12.213 & 50.324 & 0.590 & - \\
553.72 & 29.420 & 122.541 & 1.893 & - \\
246.19 & 1.412 & 6.315 & 0.093 & 0.498
\end{tabular}

M74 is a normal galaxy with no nuclear activity. Our results for this galaxy show that the FIR coming from this galaxy is from dust heated mostly by an old population. Similarly, for M83 we find that the FIR seems to be powered by old population stars.

Figure 1 shows histograms of the number of globs versus the dominating population for each galaxy. The unhatched areas represent "globs" where the heating is dominated by an old population. Figure 2 shows scatter plots of the actual fractions for each glob in each galaxy plotted versus radial distance from 


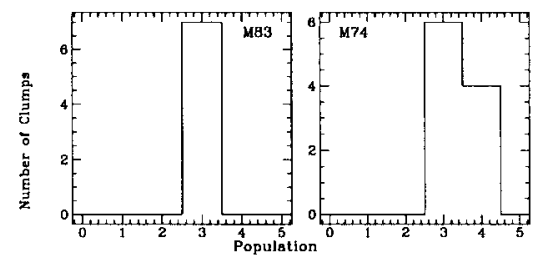

Figure 1. Above are the histograms showing that the dominating heating source for both galaxies is the old population.

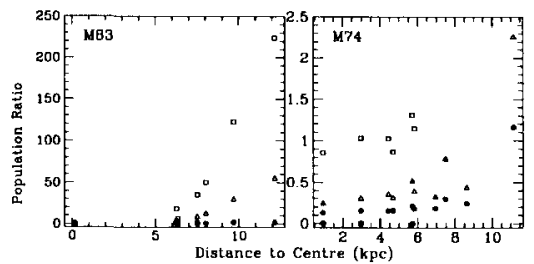

Figure 2. Above are the scatter plots showing the change in ratios with distance from the centers of the galaxies.

the nucleus. The filled circles represent the very young population, the stars are the young population, open triangles are the first old population, and open squares are the second old population.

\section{Conclusions}

We have found in this study that the bar of M83 and the quiescent nucleus of M74 seem to behave in similar ways. In both cases, the dominating heating source is an older population of stars - in both the disk and the nuclear regions. We note that other galaxies have quite different dominating populations (Jones $\&$ Keel 1995). We conclude that these two galaxies are quite similar in many ways.

Acknowledgments. Support for this paper was provided by the Alabama Space Grant Consortium and by NSF EPSCoR grant EHR-9108761. Thanks to W. Wu, S. Ryder, R. Pogge, G. Bothun and R. Bohlin for data contributions.

\section{References}

Bohlin, R. C. et al. 1983, ApJ, 274, L53

Bohlin, R. C., Cornett, R. H., \& Hill, J. K. 1990, ApJ, 363, 154

Chen, P. C. et al. 1992, ApJ, 395, L41

Cornett, R. H. et al. 1994, ApJ, 426, 553

Fitt, A. J. et al. 1992, MNRAS, 255, 146

Jones, L. V. \& Keel, W. C. 1995, ApJ, in preparation

von Hippel, T.\& Bothun, G. 1990, AJ, 100, 403 